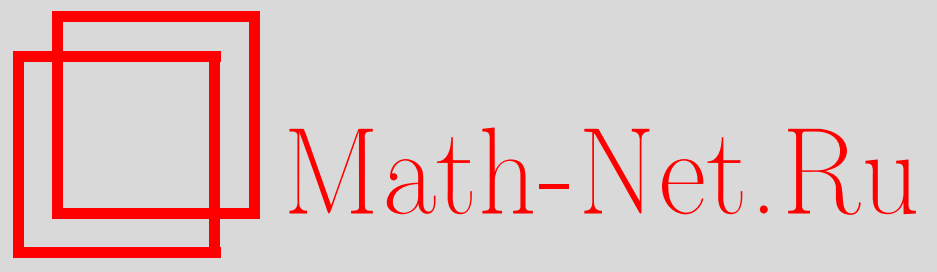

А. В. Анкилов, П. А. Вельмисов, Ю. А. Казакова, Устойчивость решений одной нелинейной начальнокраевой задачи аэроупругости, Вестн. Сам. гос. техн. ун-та. Сер. Физ.-мат. науки, 2013, выпуск 2(), 120-126

DOI: https://doi.org/10.14498/vsgtu1180

Использование Общероссийского математического портала Math-Net.Ru подразумевает, что вы прочитали и согласны с пользовательским соглашением

http://www.mathnet.ru/rus/agreement

Параметры загрузки:

IP: 35.173 .219 .149

26 апреля 2023 г., 14:01:08

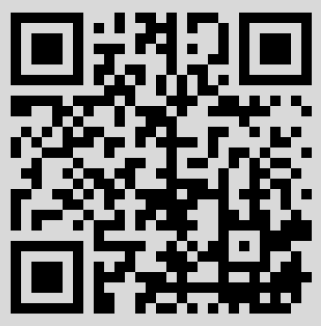


Вестн. Сам. гос. техн. ун-та. Сер. Физ.-мат. науки. 2013. № 2 (31). С. $120-126$

УДК 517.958:531.332

\title{
УСТОЙЧИВОСТЬ РЕШЕНИЙ ОДНОЙ НЕЛИНЕЙНОЙ НАЧАЛЬНО-КРАЕВОЙ ЗАДАЧИ АЭРОУПРУГОСТИ
}

\author{
А. В. Анкилов ${ }^{1}$, П. А. Вельмисов ${ }^{1}$, Ю. А. Казакова \\ 1 Ульяновский государственный технический университет, \\ Россия, 432027, Ульяновск, ул. Северный Венец, 32. \\ 2 ОАО «Ульяновское конструкторское бюро приборостроения», \\ Россия, 432071, Ульяновск, ул. Крымова, 10 А. \\ E-mails: ankil@ulstu.ru, velmisov@ulstu.ru, kazakova_ua@mail.ru
}

\begin{abstract}
Исследуется динамическая устойчивость упругого элемента стенки канала при протекании в нем дозвукового потока идеальной сжимаемой жидкости (газа). Определение устойчивости упругого тела соответствует концепции устойчивости динамических систем по Ляпунову. Получены достаточные условия устойчивости, налагающие ограничения на скорость потока, сжимающее (растягивающее) усилие, изгибную жесткость упругого элемента и другие параметры механической системы.
\end{abstract}

Ключевые слова: аэрогидроупругость, устойчивость, упругая пластина, деформация, дозвуковой поток, сжимаемая среда.

Ведение. При проектировании конструкций, обтекаемых потоком газа или жидкости, большое значение имеет исследование устойчивости деформируемых элементов, так как воздействие потока может приводить к увеличению амплитуды колебаний и тем самым к их разрушению.

В то же время для функционирования некоторых технических устройств явление возбуждения колебаний при аэрогидродинамическом воздействии, указанное выше в качестве негативного, является необходимым. Примерами подобных устройств, относящихся к вибрационной технике и используемых для интенсификации технологических процессов, являются устройства для приготовления однородных смесей и эмульсий, в частности, устройства для подачи смазочно-охлаждающей жидкости в зону обработки (см., например, Пат. 2062662 Российская Федерация, МПК6 В 06 В 1/18, 1/20. Гидродинамический излучатель / П. А. Вельмисов, Г. М. Горшков, Г. К. Рябов. Заявитель и патентообладатель Ульяновский гос. технич. ун-т. - № 5038746/28; заявл. 20.07.92; опубл. 27.06.96, Бюл. № 18).

Таким образом, при проектировании конструкций и устройств, находящихся во взаимодействии с газожидкостной средой, необходимо решать задачи, связанные с исследованием устойчивости упругих элементов, требуемой для их функционирования и надёжности эксплуатации.

1. Постановка задачи. Рассматривается плоское течение в прямолинейном канале $J=\left\{(x, y) \in \mathbb{R}^{2}: 0<x<x_{0}, 0<y<y_{0}\right\}$ (рис. 1). Часть стенки

Андрей Владимирович Анкилов (к.ф.-м.н., доц.), докторант, каф. высшей математики. Петр Александрович Велъмисов (д.ф.-м.н., проф.), профессор, каф. высшей математики. Юлия Александровна Казакова, начальник ТКБ-531. 


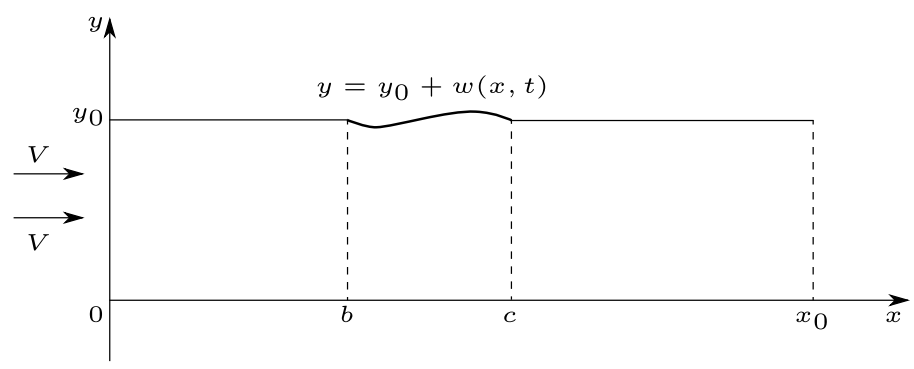

Рис. 1. Канал, стенка которого содержит деформируемый элемент

$y=y_{0}$ при $x \in[b, c]$ является упругой пластиной (упругим элементом). Скорость невозмущенного однородного потока равна $V$ и направлена вдоль оси $O x$. Используется модель идеальной сжимаемой среды. Подобные задачи для несжимаемых сред рассматривались в монографии [1].

Введём обозначения: $u(x, t)$ и $w(x, t)$ - деформации элемента в направлении осей $O x$ (продольная составляющая) и $O y$ (поперечная составляющая) соответственно; $\varphi(x, y, t)$ - потенциал скорости возмущённого потока (газа или жидкости).

Математическая постановка задачи имеет следующий вид:

$$
\begin{gathered}
\varphi_{t t}+2 V \varphi_{x t}+V^{2} \varphi_{x x}=a^{2}\left(\varphi_{x x}+\varphi_{y y}\right), \quad(x, y) \in J, \quad t \geqslant 0 \\
\varphi_{y}\left(x, y_{0}, t\right)=\dot{w}(x, t)+V w^{\prime}(x, t), \quad x \in(b, c), \quad t \geqslant 0, \\
\varphi_{y}\left(x, y_{0}, t\right)=0, \quad x \in(0, b] \cup\left[c, x_{0}\right), \quad t \geqslant 0 \\
\varphi_{y}(x, 0, t)=0, \quad x \in\left(0, x_{0}\right), \quad t \geqslant 0, \\
\varphi(0, y, t)=0, \quad \varphi\left(x_{0}, y, t\right)=0, \quad y \in\left(0, y_{0}\right), \quad t \geqslant 0 \\
\left\{\begin{array}{c}
-E F\left(u^{\prime}(x, t)+\frac{1}{2} w^{2}(x, t)\right)^{\prime}+M \ddot{u}(x, t)=0, \\
-E F\left[w^{\prime}(x, t)\left(u^{\prime}(x, t)+\frac{1}{2} w^{\prime 2}(x, t)\right)\right]^{\prime}+M \ddot{w}(x, t)+D w^{\prime \prime \prime \prime}(x, t)+ \\
+N w^{\prime \prime}(x, t)+\beta_{0} w(x, t)+\beta_{1} \dot{w}(x, t)+\beta_{2} \dot{w}^{\prime \prime \prime \prime}(x, t)= \\
=-\rho\left(\varphi_{t}\left(x, y_{0}, t\right)+V \varphi_{x}\left(x, y_{0}, t\right)\right), \quad x \in(b, c), \quad t \geqslant 0 .
\end{array}\right.
\end{gathered}
$$

Индексы $x, y, t$ снизу обозначают частные производные по $x, y, t$; штрих и точка - частные производные по $x$ и $t$ соответственно; $\rho$ - плотность жидкости в однородном невозмущенном потоке; $D, M$ - изгибная жесткость и погонная масса упругого элемента; $N$ - сжимающая (растягивающая) упругий элемент сила; $\beta_{1}, \beta_{2}$ - коэффициенты внешнего и внутреннего демпфирования; $\beta_{0}$ - коэффициент жёсткости основания; $a$-скорость звука в невозмущённом потоке жидкости $(a>V) ; E$ - модуль упругости материала элемента; $F$ - площадь поперечного сечения элемента.

Граничные условия на концах пластины при $x=b$ и $x=c$ могут иметь следующий вид:

1) жёсткое защемление:

$$
w(x, t)=w^{\prime}(x, t)=u(x, t)=0 ;
$$

2) шарнирное неподвижное закрепление:

$$
w(x, t)=w^{\prime \prime}(x, t)=u(x, t)=0 ;
$$


3) жёсткое неподвижное защемление:

$$
w(x, t)=w^{\prime}(x, t)=u^{\prime}(x, t)=0 ;
$$

4) шарнирное подвижное закрепление:

$$
w(x, t)=w^{\prime \prime}(x, t)=u^{\prime}(x, t)+\frac{1}{2} w^{\prime 2}(x, t)=0 .
$$

Уравнения и граничные условия (1)-(10) следует дополнить начальными условиями. Для трёх неизвестных функций - деформаций упругого элемента $w(x, t), u(x, t)$ и потенциала скорости сжимаемой среды $\varphi(x, y, t)$ имеет место связанная нелинейная начально-краевая задача.

2. Исследование устойчивости. Исследуем устойчивость нулевого решения $\varphi(x, y, t) \equiv 0, w(x, t) \equiv 0, u(x, t) \equiv 0$ системы $(1)-(10)$ по Ляпунову. Введём функционал

$$
\begin{gathered}
\Phi(t)=\iint_{J}\left(\varphi_{t}^{2}+\left(a^{2}-V^{2}\right) \varphi_{x}^{2}+a^{2} \varphi_{y}^{2}\right) d x d y- \\
\quad-2 a^{2} V \int_{b}^{c} \varphi\left(x, y_{0}, t\right) w^{\prime}(x, t) d x+\frac{a^{2}}{\rho} \int_{b}^{c}\left(M \dot{u}^{2}+M \dot{w}^{2}+\right. \\
\left.\quad+E F\left(u^{\prime}+\frac{1}{2} w^{\prime 2}\right)^{2}+D w^{\prime \prime 2}-N w^{\prime 2}+\beta_{0} w^{2}\right) d x .
\end{gathered}
$$

Для функций $\varphi(x, y, t), w(x, t)$ и $u(x, t)$, удовлетворяющих уравнениям $(1)$ и $(6)$, производная от $\Phi$ по $t$ примет вид

$$
\begin{aligned}
& \dot{\Phi}(t)=2 \iint_{J}\left(\varphi_{t}\left(-2 V \varphi_{x t}-V^{2} \varphi_{x x}+a^{2}\left(\varphi_{x x}+\varphi_{y y}\right)\right)+\left(a^{2}-V^{2}\right) \varphi_{x} \varphi_{x t}+\right. \\
&+\left.a^{2} \varphi_{y} \varphi_{y t}\right) d x d y-2 a^{2} V \int_{b}^{c}\left(\varphi_{t}\left(x, y_{0}, t\right) w^{\prime}(x, t)+\varphi\left(x, y_{0}, t\right) \dot{w}^{\prime}(x, t)\right) d x+ \\
&+ \frac{2 a^{2}}{\rho} \int_{b}^{c}\left(2 E F \dot{u}\left(u^{\prime}+\frac{1}{2} w^{\prime 2}\right)^{\prime}+\dot{w}\left\{-\rho\left(\varphi_{t}\left(x, y_{0}, t\right)+V \varphi_{x}\left(x, y_{0}, t\right)\right)+\right.\right. \\
&\left.+E F\left[w^{\prime}\left(u^{\prime}+\frac{1}{2} w^{\prime 2}\right)\right]^{\prime}-D w^{\prime \prime \prime \prime}-\beta_{2} \dot{w}^{\prime \prime \prime \prime}-N w^{\prime \prime}-\beta_{1} \dot{w}-\beta_{0} w\right\}+ \\
&\left.\quad+2 E F\left(u^{\prime}+\frac{1}{2} w^{\prime 2}\right)\left(\dot{u}^{\prime}+w^{\prime} \dot{w}^{\prime}\right)+D w^{\prime \prime} \dot{w}^{\prime \prime}-N w^{\prime} \dot{w}^{\prime}+\beta_{0} w \dot{w}\right) d x
\end{aligned}
$$

Произведя интегрирование с учётом условий (2)-(5), (7)-(10), получим

$$
\dot{\Phi}(t)=-\frac{2 a^{2}}{\rho} \int_{b}^{c}\left(\beta_{2} \dot{w}^{\prime \prime 2}+\beta_{1} \dot{w}^{2}\right) d x .
$$

Пусть выполняются условия

$$
\beta_{2} \geqslant 0, \quad \beta_{1} \geqslant 0, \quad \beta_{0} \geqslant 0,
$$

тогда имеют место неравенства

$$
\dot{\Phi}(t) \leqslant 0 \quad \Rightarrow \quad \Phi(t) \leqslant \Phi(0) .
$$


Для оценки функционала для функции $w(x, t)$ запишем неравенства Рэлея [2]:

$$
\begin{aligned}
\int_{b}^{c} w^{\prime \prime 2}(x, t) d x \geqslant & \lambda_{1} \int_{b}^{c} w^{\prime 2}(x, t) d x, \int_{b}^{c} w^{\prime \prime 2}(x, t) d x \geqslant \mu_{1} \int_{b}^{c} w^{2}(x, t) d x, \\
& \int_{0}^{x_{0}} \varphi_{x}^{2}(x, y, t) d x \geqslant \eta_{1} \int_{0}^{x_{0}} \varphi^{2}(x, y, t) d x
\end{aligned}
$$

где $\lambda_{1}, \mu_{1}$ - наименьшие собственные значения краевых задач

$$
\psi^{\prime \prime \prime \prime}(x)=-\lambda \psi^{\prime \prime}(x), \quad \psi^{\prime \prime \prime \prime}(x)=\mu \psi(x), \quad x \in(b, c)
$$

с граничными условиями (7)-(10); $\eta_{1}=\pi^{2} / x_{0}^{2}$ - наименьшее собственное значение краевой задачи

$$
-\psi^{\prime \prime}=\eta \psi, \quad x \in\left(0, x_{0}\right)
$$

с краевыми условиями $\psi(0)=0, \psi\left(x_{0}\right)=0$, которые соответствуют (5).

Интегрируя неравенство (15) от 0 до $y_{0}$ по переменной $y$, окончательно получим

$$
\iint_{J} \varphi_{x}^{2}(x, y, t) d x d y \geqslant \frac{\pi^{2}}{x_{0}^{2}} \iint_{J} \varphi^{2}(x, y, t) d x d y .
$$

Воспользовавшись неравенством Коши-Буняковского, получим неравенства

$$
\begin{gathered}
w^{2}(x, t) \leqslant(c-b) \int_{b}^{c} w^{2}(x, t) d x \\
\iint_{J} \varphi_{y}^{2} d x d y \geqslant \frac{2}{y_{0}^{2}} \iint_{J}\left(\varphi\left(x, y_{0}, t\right)-\varphi(x, y, t)\right)^{2} d x d y .
\end{gathered}
$$

Оценим $\Phi(0)$ сверху, используя неравенства (14) и очевидное неравенство $-2 a b \leqslant a^{2}+b^{2}$ :

$$
\begin{aligned}
\Phi(0) \leqslant \iint_{J}\left(\varphi_{t 0}^{2}\right. & \left.+\left(a^{2} V^{2}\right) \varphi_{x 0}^{2}+a^{2} \varphi_{y 0}^{2}\right) d x d y+a^{2} \int_{b}^{c} \varphi^{2}\left(x, y_{0}, 0\right) d x+ \\
+ & \frac{a^{2}}{\rho} \int_{b}^{c}\left(M \dot{u}_{0}^{2}+M \dot{w}_{0}^{2}+E F\left(u_{0}^{\prime}+\frac{1}{2} w_{0}^{\prime 2}\right)^{2}+\right. \\
& \left.+\left(D+\frac{|N|+\rho V^{2}}{\lambda_{1}}+\frac{\beta_{0}}{\mu_{1}}\right) w_{0}^{\prime \prime 2}\right) d x
\end{aligned}
$$

где введены обозначения $\varphi_{t 0}=\varphi_{t}(x, y, 0), \varphi_{x 0}=\varphi_{x}(x, y, 0), \varphi_{y 0}=\varphi_{y}(x, y, 0)$, $\dot{u}_{0}=\dot{u}(x, 0), u_{0}^{\prime}=u^{\prime}(x, 0), \dot{w}_{0}=\dot{w}(x, 0), w_{0}^{\prime}=w^{\prime}(x, 0), w_{0}^{\prime \prime}=w^{\prime \prime}(x, 0)$.

Оценим $\Phi(t)$ снизу, применяя (14), (16), (18) для (11):

$$
\begin{array}{r}
\Phi(t) \geqslant \iint_{J}\left(\varphi_{t}^{2}+\left(a^{2}-V^{2}\right) \frac{\pi^{2}}{x_{0}^{2}} \varphi^{2}+\frac{2 a^{2}}{y_{0}^{2}}\left(\varphi\left(x, y_{0}, t\right)-\varphi(x, y, t)\right)^{2}\right) d x d y- \\
-2 a^{2} V \int_{b}^{c} \varphi\left(x, y_{0}, t\right) w^{\prime}(x, t) d x+\frac{a^{2}}{\rho} \int_{b}^{c}\left(\lambda_{1} D-N\right) w^{\prime 2} d x .
\end{array}
$$


Введём обозначение

$$
f(x, t)=\left\{\begin{array}{cl}
0, & x \in(0, b] \\
w^{\prime}(x, t), & x \in(b, c), \\
0, & x \in\left[c, x_{0}\right),
\end{array}\right.
$$

тогда из (20) получим неравенство

$$
\begin{array}{r}
\Phi(t) \geqslant \iint_{J}\left[\varphi_{t}^{2}(x, y, t)+\left(\left(a^{2}-V^{2}\right) \frac{\pi^{2}}{x_{0}^{2}}+\frac{2 a^{2}}{y_{0}^{2}}\right) \varphi^{2}(x, y, t)-\right. \\
-\frac{4 a^{2}}{y_{0}^{2}} \varphi\left(x, y_{0}, t\right) \varphi(x, y, t)+\frac{2 a^{2}}{y_{0}^{2}} \varphi^{2}\left(x, y_{0}, t\right)-\frac{2 a^{2} V}{y_{0}} \varphi\left(x, y_{0}, t\right) f(x, t)+ \\
\left.\quad+\frac{a^{2}\left(\lambda_{1} D-N\right)}{\rho y_{0}} f^{2}(x, t)\right] d x d y .
\end{array}
$$

Согласно критерию Сильвестра квадратичная форма относительно $\varphi(x, y, t)$, $\varphi\left(x, y_{0}, t\right), f(x, t)$ в $(21)$ будет положительно определенной, если выполняются условия

$$
\begin{gathered}
\lambda_{1} D-N>0 \\
\frac{\lambda_{1} D-N}{\rho y_{0}} \cdot \frac{2\left(a^{2}-V^{2}\right) \pi^{2}}{x_{0}^{2}}-V^{2}\left(\frac{\left(a^{2}-V^{2}\right) \pi^{2}}{x_{0}^{2}}+\frac{2 a^{2}}{y_{0}^{2}}\right)>0 .
\end{gathered}
$$

Преобразуем неравенство (23):

$$
N<\lambda_{1} D-\frac{V^{2} x_{0}^{2} \rho y_{0}}{2\left(a^{2}-V^{2}\right) \pi^{2}}\left(\frac{\left(a^{2}-V^{2}\right) \pi^{2}}{x_{0}^{2}}+\frac{2 a^{2}}{y_{0}^{2}}\right) .
$$

Оценивая квадратичную форму в $(21)$ относительно $w(x, t)$ с учётом $(17)$, получим

$$
\Phi(t) \geqslant \frac{\Delta_{3} y_{0}}{\Delta_{2}(c-b)} w^{2}(x, t)
$$

где

$$
\begin{gathered}
\Delta_{2}=d_{11} d_{22}-d_{12}^{2}>0, \Delta_{3}=d_{33} \Delta_{2}-d_{23}^{2} d_{11}>0, d_{11}=\frac{\left(a^{2}-V^{2}\right) \pi^{2}}{x_{0}^{2}}+\frac{2 a^{2}}{y_{0}^{2}}, \\
d_{22}=d_{12}=\frac{2 a^{2}}{y_{0}^{2}}, d_{23}=\frac{V}{y_{0}^{2}}, d_{33}=\frac{a^{2}\left(\lambda_{1} D-N\right)}{\rho y_{0}} .
\end{gathered}
$$

Учитывая (13), (19), (25), получим неравенство

$$
\begin{aligned}
& w^{2}(x, t) \leqslant \frac{\Delta_{2}(c-b)}{\Delta_{3} y_{0}}\left[\iint_{J}\left(\varphi_{t 0}^{2}+\left(a^{2}-V^{2}\right) \varphi_{x 0}^{2}+a^{2} \varphi_{y 0}^{2}\right) d x d y+\right. \\
&+a^{2} \int_{b}^{c} \varphi^{2}\left(x, y_{0}, 0\right) d x+\frac{a^{2}}{\rho} \int_{b}^{c}\left(M \dot{u}_{0}^{2}+M \dot{w}_{0}^{2}+E F\left(u_{0}^{\prime}+\frac{1}{2} w_{0}^{\prime 2}\right)^{2}+\right. \\
&\left.\left.+\left(D+\frac{|N|+\rho V^{2}}{\lambda_{1}}+\frac{\beta_{0}}{\mu_{1}}\right) w_{0}^{\prime \prime 2}\right) d x\right] .
\end{aligned}
$$


Теорема 1. Пусть выполняются условия (12), (22), (24). Тогда решение $w(x, t)$ задачи (1)-(10) устойчиво по отношению $\kappa$ возмущениям начальньх данных $\varphi_{t 0}, \varphi_{x 0}, \varphi_{y 0}, \varphi\left(x, y_{0}, 0\right), \dot{u}_{0}, u_{0}^{\prime}, \dot{w}_{0}, w_{0}^{\prime}, w_{0}^{\prime \prime}$.

Аналогично, оценивая квадратичную форму в $(21)$ относительно $\varphi(x, y, t)$, получим

$$
\Phi(t) \geqslant \frac{\Delta_{3}}{d_{22} d_{33}-d_{23}^{2}} \iint_{J} \varphi^{2}(x, y, t) d x d y .
$$

Учитывая (13), (19), (26), получим неравенство

$$
\begin{array}{r}
\iint_{J} \varphi^{2}(x, y, t) d x d y \leqslant \frac{d_{22} d_{33}-d_{23}^{2}}{\Delta_{3}}\left[\iint_{J}\left(\varphi_{t 0}^{2}+\left(a^{2}-V^{2}\right) \varphi_{x 0}^{2}+a^{2} \varphi_{y 0}^{2}\right) d x d y+\right. \\
+a^{2} \int_{b}^{c} \varphi^{2}\left(x, y_{0}, 0\right) d x+\frac{a^{2}}{\rho} \int_{b}^{c}\left(M \dot{u}_{0}^{2}+M \dot{w}_{0}^{2}+E F\left(u_{0}^{\prime}+\frac{1}{2} w_{0}^{\prime 2}\right)^{2}+\right. \\
\left.\left.+\left(D+\frac{|N|+\rho V^{2}}{\lambda_{1}}+\frac{\beta_{0}}{\mu_{1}}\right) w_{0}^{\prime \prime 2}\right) d x\right] .
\end{array}
$$

Теорема 2. Пусть выполняются условия (12), (22), (24). Тогда решение $\varphi(x, y, t)$ задачи (1)-(10) устойчиво в среднем (в интегральном смысле) по отношению $\kappa$ возмущениям начальных данных $\varphi_{t 0}, \varphi_{x 0}, \varphi_{y 0}, \varphi\left(x, y_{0}, 0\right), \dot{u}_{0}$, $u_{0}^{\prime}, \dot{w}_{0}, w_{0}^{\prime}, w_{0}^{\prime \prime}$.

3. Пример механической системы. Рабочая среда - воздух $(\rho=1)$, пластина изготовлена из алюминия $\left(E=7 \cdot 10^{10}, \rho_{p l}=8480\right)$. Другие параметры механической системы: $a=331, x_{0}=5, y_{0}=0,1, b=2, c=3, h=0,005, \nu=0,31$, $D=\frac{E h^{3}}{12\left(1-\nu^{2}\right)}=806,7$. Пусть концы упругой пластины закреплены шарнирно, тогда $\lambda_{1}=\pi^{2} /(c-b)^{2}=\pi^{2}$. Все значения приведены в системе СИ.

Для неравенства (24) построены области устойчивости на плоскости «сжимающее (растягивающее) усилие $N$ - скорость потока $V »$ (рис. 2 ).

На рис. 2 серая область - область устойчивости. Прямая $V=a$ является асимптотой границы области (24).
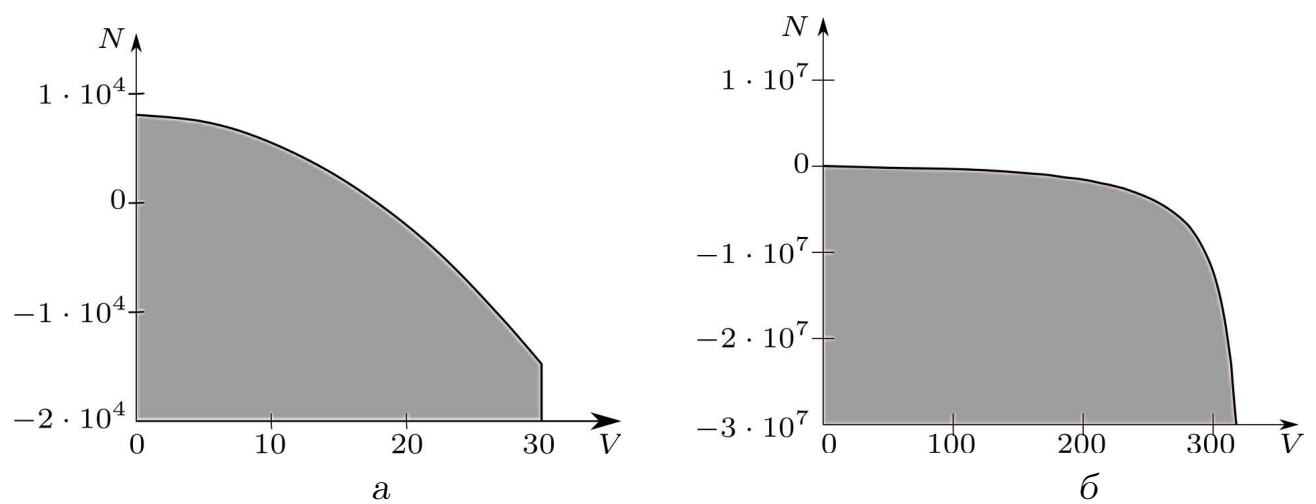

Рис. 2. Области устойчивости 
Работа выполнена в рамках государственного задания Министерства образования и науки РФ (НИР 1.632.2011).

\title{
БИБЛИОГРАФИЧЕСКИЙ СПИСОК
}

1. А. В. Анкилов, П. А. Велъмисов, Устойчивость вязкоупругих элементов стенок проточных каналов. Ульяновск: Ульяновский гос. технич. ун-т, 2000. 115 с. [A. V. Ankilov, P. A. Vel'misov, Stability of viscoelastic elements of channels walls. Ulyanovsk: Ulyanovsk Technical University, 2000. 115 pp.]

2. Л. Коллати, Задачи на собственные значения. М.: Наука, 1968. 503 с. [L. Kollatc, Problems on own values. Moscow: Nauka, 1968. 503 pp.]

Поступила в редакцию 16/XI/2012; в окончательном варианте - 27/III/2013.

MSC: 74F10; 35Q35, 35Q74

STABILITY OF SOLUTION OF ONE NONLINEAR INITIAL-BOUNDARY PROBLEM OF AEROELASTICITY

\author{
A. V. Ankilov ${ }^{1}$, P. A. Vel'misov ${ }^{1}$, Yu. A. Kazakova ${ }^{2}$ \\ 1 Ulyanovsk State Technical University, \\ 32, Severny Venets st., Ulyanovsk, 432027, Russia. \\ 2 Ulyanovsk Instrument Manufacturing Design Bureau, \\ 10 A, Krymov st., Ulyanovsk, 432071, Russia. \\ E-mails: ankil@ulstu.ru, velmisov@ulstu.ru, kazakova_ua@mail.ru
}

The dynamic stability of an elastic element of the channel wall under the subsonic stream of an ideal compressible fluid (gas) is studied. Determination of the stability of an elastic body corresponds to the concept of stability of dynamical systems by Lyapunov. The sufficient conditions for stability are obtained. Conditions impose limitations on the speed of the uniform stream of gas, compressed (tensile) element of efforts, the elastic element stiffness and other parameters of the mechanical system.

Key words: aerohydroelasticity, stability, elastic plate, deformation, subsonic flow, compressed liquid (gas).

Original article submitted 16/XI/2012; revision submitted $27 / \mathrm{III} / 2013$.

Andrey V. Ankilov (Ph. D. (Phys. \& Math.)), Doctoral Candidate, Dept. of Higher Mathematics. Petr A. Vel'misov (Dr. Sci. (Phys. \& Math.)), Professor, Dept. of Higher Mathematics. Yulia A. Kazakova, Chief of TKB-531. 